\title{
Planar Crossing Numbers of Genus $g$ Graphs
}

\author{
Hristo Djidjev ${ }^{1 \star}$, Imrich Vrt'o ${ }^{2} \star$ \\ 1 Los Alamos National Laboratory \\ P.O. Box 1663, Los Alamos, NM 87545, USA \\ 2 Institute of Mathematics, Slovak Academy of Sciences \\ Dúbravská 9, 84104 Bratislava, Slovak Republic \\ djidjev@lanl.gov, vrto@savba.sk
}

\begin{abstract}
Pach and Tóth [15] proved that any $n$-vertex graph of genus $g$ and maximum degree $d$ has a planar crossing number at most $c^{g} d n$, for a constant $c>1$. We improve on this results by decreasing the bound to $O(d g n)$, if $g=o(n)$, and to $O\left(g^{2}\right)$, otherwise, and also prove that our result is tight within a constant factor.
\end{abstract}

\section{Introduction}

A drawing of a graph $G$ in the plane is an injection of the set of the vertices of $G$ into points of the plane and a mapping of the set of the edges of $G$ into simple continuous curves such that the endpoints of each edge are mapped onto the endpoints of its image curve. Moreover, no curve should contain an image of a vertex in its inside and no three curves should intersect in the same point, unless it is an endpoint. The planar crossing number (or simply the crossing number) of $G$, denoted by $\operatorname{cr}(G)$, is the minimum number of edge crossings over all drawings of $G$ in the plane.

The concept of crossing numbers was introduced by Turán [20] more than 50 years ago. Although there have been scores of results and publications since, because of the difficulty of the problem there are only a few infinite classes of graphs with determined exact crossing numbers. For instance, Glebsky and Salazar recently proved that the crossing number of the Cartesian product of two cycles $C_{m} \times C_{n}$ is $(m-2) n[11]$. But the exact crossing numbers for such important graphs as the complete graph $K_{m}$ and the bipartite graph $K_{m, n}$ are not known. For an annotated bibliography of crossing number results see [14] and for a more extensive and up-to-date chronological bibliography see [19].

From algorithmic point of view, crossing numbers have been studied by Leighton [13], who was motivated by their application in VLSI design. In graph drawing, crossing numbers have been used for finding aesthetic drawing of nonplanar graphs and graph-like structures [3]. Typically, such graphs are drawn in the plane with a small number of crossings and next each crossing point is replaced by a new vertex of degree 4 . The resulting planar graph is then drawn in the plane using an existing algorithm for nicely drawing a planar graph, and finally the new vertices are removed and replaced back by edge crossings. The general drawing heuristics are usually based on the divide and conquer approach, using good separators, or using 2-page layouts $[4,13]$.

The problem of finding the crossing number of a given graph was first proved to be NP-hard by Garey and Johnson [9] and, more recently, it was shown to be NP-hard even for cubic graphs [12]. There is only one exact algorithm of practical use [2], but it works for small and sparse graphs only. The best polynomial algorithm approximates the crossing number with a polylogarithmic factor [8].

Another direction of research is to estimate crossing numbers in terms of basic graph parameters, like density and edge separators. There are only a few results of this type [1,13]. And although the crossing number and the genus of the graph are two of the most important measures for nonplanarity, there are only a few results that study the relationship between them. Pach and Tóth [15] showed that

\footnotetext{
* This work has been supported by the Department of Energy under contract W-705-ENG-36 and by the Los Alamos National Laboratory LDRD-DR Grant "Statistical Physics of Infrastucture Networks"

** This research was partially supported by the VEGA grant No. 2/6089/26 and the EPSRC grant GR/S76694/01.
} 
any $n$-vertex $d$-degree toroidal graph $G$ (i.e., graph that can be drawn on the torus with no intersections) has crossing number $O(d n)$. If $G$ is of genus $g$ (i.e., can be drawn on a surface $S_{g}$ of genus $g$ with no intersections), they proved that

$$
\operatorname{cr}(G) \leq c^{g} d n,
$$

for some constant $c>1$. Unfortunately, the constant $c$ is very large and, as a consequence, their result can be useful for very small values of $g$ only. Although their proofs are of a constructive type, Pach and Tóth do not discuss algorithmic issues.

In this paper we show that

$$
\begin{array}{ll}
\operatorname{cr}(G)=O(d g n), & \text { for } g=o(n), \quad \text { or } \\
\operatorname{cr}(G)=O\left(g^{2}\right), & \text { otherwise, }
\end{array}
$$

and that the bound is tight within a constant factor. Our approach allows one to estimate the surface $g^{\prime}$ crossing numbers of genus $g$ graphs drawn on any surface $S_{g^{\prime}}$ of genus $g^{\prime}$, for $g^{\prime}<g$.

Our result is also interesting because of the fact that it relates the crossing numbers of a given graph on two different surfaces. Specifically, let $\mathrm{cr}_{g}(G)$ denote the surface $g$ crossing number of $G$, i.e., the minimum number of edge crossings over all drawings of $G$ in $S_{g}$. The above type of results says that if $\mathrm{cr}_{g}(G)=0$, then $\operatorname{cr}(G)$ cannot be very large. We further strengthen this result by showing as a corollary of our main result (1) that $\operatorname{cr}(G)=O\left(\operatorname{cr}_{g}(G) g+g n\right)$ for bounded degree graphs.

This paper is organized as follows. In Section 2 we give some basic definitions and facts about embeddings and surfaces. In Section 3, we prove our main result (1) and describe the drawing algorithm based on our upper bound proof. In Section 4, we give a lower bound proof that shows the tightness of (1). We conclude with a discussion of extensions and generalizations of the results presented in the paper.

\section{Preliminaries}

In this paper by $G$ we denote an undirected graph and by $V(G)$ and $E(G)$ we denote the set of the vertices and the set of the edges of $G$, respectively. The size of $G$ is $|G|=|V(G)|+|E(G)|$. For any vertex $v$, the number of the adjacent vertices to $v$ is called the degree of $v$ and is denoted by $\operatorname{deg}(v)$. The maximum degree of any vertex of $G$ is called the degree of $G$. The set of the vertices adjacent to $v$ is called the neighborhood of $v$ and is denoted by $N(v)$. For any set of vertices $X$ the neighborhood of $X$ is $N(X)=\bigcup_{v \in X} N(v)$.

The bisection width of $G$, denoted by bw $(G)$, is the smallest number of edges whose removal divides the graph into parts having no more than $2|V(G)| / 3$ vertices each.

By a surface we mean a closed manifold and by $S_{g}$ we denote a surface of genus $g$. A drawing of $G$ on $S_{g}$ is any injection of the vertices of $G$ onto points of $S_{g}$ and the edges of $G$ onto continuous simple curves of $S_{g}$ so that the endpoints of any edge are mapped onto the endpoints of its corresponding curve. The drawing is called an embedding, if no two curves intersect, except possibly at an endpoint. The genus of $G$, denoted by $g(G)$, is the smallest genus of a surface $G$ can be embedded in. $G$ is planar, if the genus of $G$ is zero. Every planar graph can be drawn in the plane without any edge intersections. Throughout this paper we will use combinatorial representations of embeddings, where each undirected edge of $G$ is replaced by a pair of opposite directed edges, and the cyclic list of outgoing edges from any vertex $v$ (called edge-orbit) specifies the counterclockwise order in which the edges appear around $v$ in the embedding. In a facial walk, the successor of any edge $(v, w)$ is the edge after $(w, v)$ in the edge-orbit for $w$. The faces of embedding are all simple closed facial walks and they correspond to the maximal connected regions into which the drawing of $G$ divides the plane. The outer face of a planar embedding corresponds to the infinite face of the corresponding drawing. In a combinatorial embedding, any face can be chosen to be the outer face.

In the remainder of this paper, we will use $n, m$, and $d$ to denote the numbers of vertices, edges, and the degree of $G$, respectively. We also assume that we are given an embeding $\mu(G)$ of $G$ in $S_{g}$ as an input. If $f$ denotes the number of the faces of $\mu(G)$, then the Euler characteristic $\mathcal{E}(\mu(G))$ of $\mu(G)$, denoted simply by $\mathcal{E}(G)$ when the embedding is clear from the context, is defined as

$$
\mathcal{E}(G)=\mathcal{E}(\mu(G))=n-m+f .
$$


The relation between the Euler characteristic and the genus $g$ of the embedding is given by the Euler formula

$$
n-m+f=2-2 g .
$$

For any subgraph $K$ of $G$, let $\mu(K)$ denote the embedding of $K$ induced by $\mu(G)$, let $g_{\mu}(K)$ denote the genus of $\mu(K)$, and let $g(K)$ denote the genus of $K$. Note that $g_{\mu}(K)$ and $g(K)$ may not be equal. In order to simplify notations, we denote $g_{K}=g_{\mu}(K)$.

\section{The drawing algorithm}

We will start in Section 3.1 by describing a procedure for partitioning $G$ into components with special properties, which we divide into three classes. In Section 3.2 we will outline the rest of the algorithm that draws each component according to its type and then combines all drawings into a drawing of the original graph.

\subsection{Dividing the graph into components.}

Without a loss of generality we assume that $G$ is biconnected, since otherwise one can draw the biconnected components separately in the plane and then combine their drawings into a planar drawing of $G$. Triangulate $\mu(G)$ by inserting a suitable number of additional edges in each face that is not a triangle. Assign weights 1 to all original edges of $G$ and weights 0 to all new edges.

In order to simplify the notations, we will continue to denote by $G$ and $\mu(G)$ the modified graph and embedding, respectively, and will refer to the edges of weights 1 and 0 as original and new edges of $G$, respectively. For any set $X \subseteq V(G)$, let $w t(X)$ denote the sum of the weights of all edges of $X$. Since in our algorithms we will only be interested in intersections between original edges of $G$, we introduce the term original crossings to refer to crossings where both intersecting edges are original.

Select any vertex $t$ and divide the vertices of $G$ into levels according to their distance to $t$. For a constant $r$ to be determined later, denote by $L_{j}$, for $0 \leq j<r$, the set of all edges between level $i$ and level $i+1$ vertices, for all $i$ satisfying $i \bmod r=j$. Assume that the number of all levels is at least $r$. Then there exists an $i^{*}<r$ such that

$$
w t\left(L_{i^{*}}\right) \leq\lfloor m / r\rfloor .
$$

Replace each edge $e=(v, w) \in L_{i^{*}}$ by a pair of new edges $s_{1}=\left(v, x_{1}\right)$ and $s_{2}=\left(w, x_{2}\right)$ called stubs, where $x_{1}$ and $x_{2}$ are new vertices. This has the effect of "cutting" $e$. The stubs $s_{1}$ and $s_{2}$ are a matching pair of stubs and $e$ is a parent of $s_{1}$ and $s_{2}$. For any stub $(v, x)$, where $v \in V(G)$ and $x \notin V(G)$, vertex $v$ is called attached and vertex $x$ is called unattached. Our drawing algorithm will eventually join each pair of stubs back into their parent edge.

Compute the connected components of the resulting graph, $G^{\prime}$. For any component $K$ of $G^{\prime}$, let $q_{K}$ and $q_{K}^{\prime}$ denote the number of the bicomponents induced by the vertices on the lowest and on the highest level of $K$, respectively, that are incident to edges from $L_{i^{*}}$. Without loss of generality, we assume that $q_{K}>0$ for any component $K$ except the one containing $t$, since otherwise $K$ will be degenerate (a forest), which is easy to draw without intersections. Denote $L_{K}=N(V(K)) \cap L_{i^{*}}$.

Let $\mu(K)$ be the embedding of $K$ induced by the embedding of $G$, let $n_{K}$ and $m_{K}$ be the numbers of the vertices and the edges of $K$, and let $f_{K}$ be the number of the faces of $\mu(G)$ whose all edges are in $K$.

Lemma 1. The Euler characteristic of $\mu(K)$ is

$$
\mathcal{E}(K)=n_{K}-m_{K}+f_{K}+q_{K}+q_{K}^{\prime} .
$$

Proof. Denote by $\mathcal{K}(K)$ the set of bicomponents induced by the vertices on the lowest and the highest levels of $K$. Then $\mu(K)$ has exactly one new face for each bicomponent of $\mathcal{K}(K)$. The lemma follows from (2).

Corollary 1. The embedding $\mu(K)$ has genus $g_{K}=1-\left(n_{K}-m_{K}+f_{K}+q_{K}+q_{K}^{\prime}\right) / 2$. 
Assign a label $\left(q_{K}, q_{K}^{\prime}\right)$ to $K$. Consider the set $M$ of all components $K$ with $g_{K}=0$ (i.e., such that $\mu(K)$ is a planar embedding) and with label $(1,1)$ or $(1,0)$. Merge any two components $K_{1}$ and $K_{2}$ from $M$ that contain a pair of matching stubs by replacing all pairs of matching stubs by their parent edges. Assign a label $(1,1)$ to the resulting component, $\bar{K}$, and continue until no more merges are possible.

Let $\mathcal{K}(G)$ denote the set of all resulting components of $G$. We will use the following lemma from [7].

Lemma 2. Let $q_{G^{\prime}}$ denote the sum of $q_{K}$ over all components $K$ of $G^{\prime}$. Then

$$
g_{\mu}\left(G^{\prime}\right) \leq g(G)-q_{G^{\prime}}+|\mathcal{K}(G)|-1
$$

The next lemma shows that, after the merges, the number of the resulting components is $O(g)$.

Lemma 3. $|\mathcal{K}(G)|<2 g$.

Proof. Since $g_{\mu}\left(G^{\prime}\right) \geq 0$, by (4)

$$
q_{G^{\prime}}-|\mathcal{K}(G)|<g(G)=g
$$

Let $\mathcal{K}^{\prime}$ be the set of all components $K$ with $q_{K}=1$ and let $\mathcal{K}^{\prime \prime}$ be the set of components $K$ with $q_{K}>1$. Let $q^{\prime}$ and $q^{\prime \prime}$ be the sums of $q_{K}$ for $K$ in $\mathcal{K}^{\prime}$ and $\mathcal{K}^{\prime \prime}$, respectively. Then clearly $q^{\prime}=\left|\mathcal{K}^{\prime}\right|$, $q^{\prime}+q^{\prime \prime}=q_{G^{\prime}}$, and $|\mathcal{K}(G)|=\left|\mathcal{K}^{\prime}\right|+\left|\mathcal{K}^{\prime \prime}\right|$. By $(5), q^{\prime \prime}-\left|\mathcal{K}^{\prime \prime}\right|<g$. Since each component in $\mathcal{K}^{\prime \prime}$ contributes to the sum $q^{\prime \prime}$ with at least 2 , then $q^{\prime \prime} \geq 2\left|\mathcal{K}^{\prime \prime}\right|$ and hence $\left|\mathcal{K}^{\prime \prime}\right|<g$. The claim follows as $\left|\mathcal{K}^{\prime}\right| \leq\left|\mathcal{K}^{\prime \prime}\right|$.

The next result strengthens Lemma 3 by showing that not only the number of the components of $G^{\prime}$, but also the sum of their labels is $O(g)$.

Lemma 4. $\sum_{K \in \mathcal{K}(G)}\left(q_{K}+q_{K}^{\prime}\right)<6 g$.

Proof. For each bicomponent induced by the vertices on level $i$ and incident to edges from $L_{i^{*}}$, there corresponds at least one bicomponent induced by the vertices on level $i+1$, where $i \bmod r=i^{*}$. Then we have

$$
q_{G^{\prime}}=\sum_{K \in \mathcal{K}(G)} q_{K} \geq \sum_{K \in \mathcal{K}(G)} q_{K}^{\prime}
$$

By (4),

$$
q_{G^{\prime}}-|\mathcal{K}(G)|+1 \leq g(G)-g_{\mu}\left(G^{\prime}\right) \leq g(G)=g .
$$

Combining the last inequality with Lemma 3 gives

$$
q_{G^{\prime}} \leq|\mathcal{K}(G)|+g-1<3 g .
$$

The lemma follows by (6).

We will divide all components $K$ of $G^{\prime}$ into three classes depending on their labels $\left(q_{K}, q_{K}^{\prime}\right)$ and on $g_{K}$ as follows. (i) If $g_{K}>0$, then $K$ will be of non-planar type (note that $K$ can actually be planar if the genus of $K$ is smaller than the genus of $\mu(K)$ ). (ii) If $g_{K}=0$ and the label of $K$ is $(1,1)$ or $(1,0)$, then $K$ will be of l-planar (for "long planar") type. (iii) If $g_{K}=0$ and the label of $K$ is not $(1,1)$ or $(1,0)$, then $K$ will be of s-planar (for "short planar") type. Recall that components of non-planar and s-planar types have no more than $r$ levels, but components of l-planar type can have larger number of levels.

\subsection{Algorithm outline}

The rest of the algorithm draws each component $K$ of $G^{\prime}$ in the plane according to its type. The goal is to have the unattached endpoints of all stubs drawn in the outer face and a relatively small number of original crossings between edges of $K$. After all components are drawn in this way, all pairs of matching stubs are joined into their parent edges. As all stubs are already in the outer face, intersections may occur only between pairs of stubs. Since, by (3), the weight of all stubs is $O(m / r)$, this final step will increase the total number of original crossings by $O\left((\mathrm{~m} / \mathrm{r})^{2}\right)$. 


\subsection{Drawing non-planar components}

If $K$ is non-planar, then we will show that a subgraph of $K$ of relatively small size can be found such that "cutting" the embedding of $K$ along the edges of that subgraph and appropriately pasting a face $f$ along the cut produces a planar surface. Then we will draw $K$ in the plane with $f$ as an outer face and redraw the edges that were destroyed by the cut. Since those edges will be entirely in $f$, they will not intersect other edges of $K$. Finally, we will route all stubs to the outer face.

3.3.1 Finding a planarizing set for $\boldsymbol{K}$. Consider a component $K$ such that $g_{K}>0$ and let $l_{K}^{-}$and $l_{K}^{+}$denote the lowest and the highest levels of $K$. Define a spanning forest $F_{K}$ of $K$ with $q_{K}$ trees as follows. For each connected bicomponent $Q$ defined by the vertices of $K$ on level $l_{K}^{-}$define a spanning tree $T_{Q}$ for $Q$ and add the edges of $T_{Q}$ to $F_{K}$. For each vertex $v$ on level greater than $l_{K}^{-}$choose any vertex $w$ on a lower level adjacent to $v$ and add edge $(v, w)$ to $F_{K}$. Finally, for each stub $s$ from $K$, make the attached endpoint of $s$ parent of its unattached endpoint. Clearly, $F_{K}$ contains $q_{K}$ trees, one for each connected bicomponent induced by level $l_{K}^{-}$in $K$.

We will call an $F_{K}$-cycle any simple cycle in $K$ that has exactly one non-forest edge. Since $K$ has no-more than $r$ levels, any $F_{K}$-cycle will contain no more than $2(r-1)$ vertices of $K$, excluding the vertices on level $l_{K}^{-}$.

For any non-forest edge $e$ of $K$ incident to two different faces $f_{1}$ and $f_{2}$ of the embedding, remove $e$ and merge $f_{1}$ and $f_{2}$ into a single face. Since this operation eliminates one edge and one face, the Euler characteristic does not change. Continue until no such edge $e$ remains. Then any of the remaining nonforest edges should be incident only to $f$. Clearly, $f$ should be the only face of the resulting embedding, since any face must contains a non-forest edge (otherwise $T$ will contain a cycle). Next, remove any edge that is incident to a degree- 1 vertex as well as the degree- 1 vertex itself. Since each removal reduces the number of the vertices and the number of the edges by one, this operation preserves the Euler characteristic of $\mu(K)$.

Denote by $P l(K)$ the resulting graph. We will think of $P l(K)$ as a "planarizing" graph since, as we will show in Step 4.2, it can be used to transform the embedding of $K$ into a planar embedding. Denote by $n_{P l}$ and $m_{P l}$ the number of the vertices and the number of the edges of $P l(K)$. By (2) we have

$$
n_{P l}-m_{P l}+1=2-2 g_{K},
$$

and hence

$$
m_{P l}=\left(n_{P l}-1\right)+2 g_{K},
$$

which implies that the number of remaining non-forest edges is $2 g_{K}$. Therefore, $P l(K)$ is a union of $2 g_{K} F_{K}$-cycles.

We proved the following.

Lemma 5. The embedding $\mu(P l(K))$ of $P l(K)$ has a single face, genus $g_{K}$, and no more than $2 g_{K}(r-1)$ vertices whose levels are in the interval $\left(l_{K}^{-}, l_{K}^{+}\right]$.

We will use $P l(K)$ in the next subsection to "planarize" $\mu(K)$.

3.3.2 Transforming $\boldsymbol{\mu}(\boldsymbol{K})$ into a planar embedding. Next we transform $\mu(K)$ by modifying $P l(K)$ so that it is transformed into a new face $f$ bounded by a simple cycle $c$. See the example on Figure 1 (b). Next we describe more formally the transformation of the different elements of $K$. We denote by $e=\left(w_{1}, w_{2}\right)$ an edge of $K$ with at least one endpoint in $\operatorname{Pl}(K)$.

1) Vertices of $P l(K)$. Let $v$ be any vertex of degree $k$ from $P l(K)$ and let $\left\langle e_{1}, \cdots, e_{k}\right\rangle$ be the counterclockwise permutation of the edges of $P l(K)$ incident to $v$. Define $k$ new vertices that will replace $v$ and denote them by $\left\{e_{1}, e_{2}\right\},\left\{e_{2}, e_{3}\right\}, \cdots,\left\{e_{k}, e_{1}\right\}$ (Figure 1 (a)).

2) Edges not in $\mathrm{Pl}(K)$. Let $e \notin E(P l(K))$. We will define an edge $\left(w_{1}^{\prime}, w_{2}^{\prime}\right)$ to replace $e$. If $w_{1}$ is not from $P l(K)$ let $w_{1}^{\prime}=w_{1}$. Else denote by $\left\langle e_{1}, \cdots, e_{k}=e_{0}>\right.$ the edge-orbit of $w_{1}$ and let $e_{j}$ be the first edge from $P l(K)$ in a counterclockwise direction from $e$ and let $e_{j-1}$ be the first edge from $P l(K)$ 


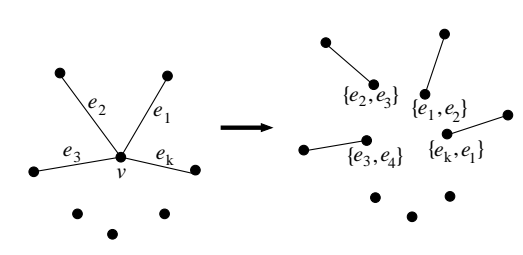

(a)

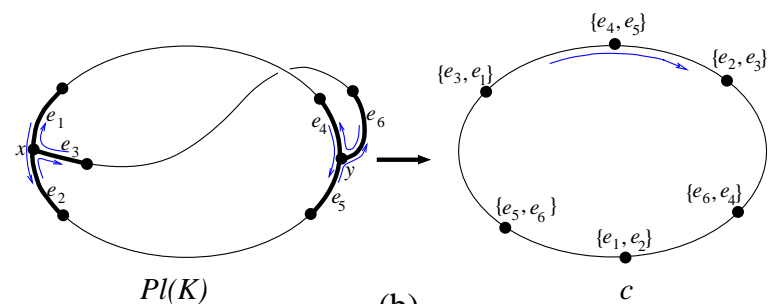

(b)

Fig. 1. The transformation of $P l(K)$. (a) Replacing $v$ by $k$ new vertices. (b) Replacing $P l(K)$ by a simple cycle $c$. The arrows show the direction of the face walk.

in a clockwise direction. Then define $w_{1}^{\prime}=\left\{e_{i}, e_{j}\right\}$. Similarly define a vertex $w_{2}^{\prime}$ corresponding to $w_{2}$. Replace $e=\left(w_{1}, w_{2}\right)$ by the edge $\left(w_{1}^{\prime}, w_{2}^{\prime}\right)$, which we will denote by new $\left(w_{1}, w_{2}\right)$.

3) Edges from $P l(K)$. Let $e \in E(P l(K))$ and let $e^{\prime}$ be the first edge from $P l(K)$ in a clockwise direction from $e$ in the edge-orbit of $w_{2}$ and let $e^{\prime \prime}$ be the first edge from $P l(K)$ in a counterclockwise direction of the edge-orbit of $w_{1}$. Define a new edge $\overrightarrow{n e w}\left(w_{2}, w_{1}\right)=\left(\left\{e, e^{\prime \prime}\right\},\left\{e^{\prime}, e\right\}\right)$. Similarly, define an edge $\overrightarrow{n e w}\left(w_{1}, w_{2}\right)$ by swapping $w_{1}$ and $w_{2}$. Finally, replace $e$ by the two edges $\overrightarrow{n e w}\left(w_{2}, w_{1}\right)$ and $\overrightarrow{n e w}\left(w_{1}, w_{2}\right)$ (note that both those new edges are undirected).

4) Updating the edge-orbits. Next update the edge-orbits for the vertices incident to the new edges as follows. Let $w$ be a vertex of $\operatorname{Pl}(K)$ and let $<e_{1}=\left(w, v_{1}\right), \cdots, e_{k}=\left(w, v_{k}\right)>$ be the counterclockwise permutation of the edges of $K$ incident to $w$. For any pair of edges $\left(w, v_{i}\right)$ and $\left(w, v_{j}\right), 1 \leq i, j \leq k$, such that $\left(w, v_{j}\right)$ is the first edge from $P l(K)$ in a counterclockwise direction from $\left(w, v_{i}\right)$ define the edge-orbit of the new vertex $w\left(e_{i}, e_{j}\right)$ as follows: $<\overrightarrow{n e w}\left(w, v_{i}\right), n e w\left(w, v_{i+1}\right), \cdots, n e w\left(w, v_{j-1}\right), \overrightarrow{n e w}\left(v_{j}, w\right)>$.

Denote by $K^{\prime}$ the resulting component, by $\bar{\mu}\left(K^{\prime}\right)$ its embedding, and by $c$ the cycle corresponding to $\mathrm{Pl}(K)$..

In order to simplify notations, let $V_{P l}=V(P l(K)), V_{c}=V(c)$. By construction, we have the following.

Lemma 6. The resulting component $K^{\prime}$, its embedding $\bar{\mu}\left(K^{\prime}\right)$, and the cycle c constructed by the transformation of $\mathrm{Pl}(K)$ have the following properties:

(a) $V(K)=V\left(K^{\prime}\right) \backslash V_{c} \cup V_{P l}, E(K)=E\left(K^{\prime}\right) \backslash N\left(V_{c}\right) \cup N\left(V_{P l}\right)$;

(b) $\left\{N(v) \mid v \in V_{P l}\right\} \backslash V_{P l}=\left\{N(v) \mid v \in V_{c}\right\} \backslash V_{c}$;

(c) The number of the faces of $\bar{\mu}\left(K^{\prime}\right)$ exceeds the number of the faces of $\mu(K)$ by one.

Lemma 7. The embedding $\bar{\mu}\left(K^{\prime}\right)$ is planar.

Proof. Let $n_{K}, m_{K}$, and $f_{K}$ be the number of the vertices, edges, and faces of $\mu(K)$ and let $n_{K}^{\prime}, m_{K}^{\prime}$, and $f_{K}^{\prime}$ be those numbers for $\bar{\mu}\left(K^{\prime}\right)$. Since the genus of $\mu(K)$ is $g_{K}$, by $(2)$

$$
n_{K}-m_{K}+f_{K}=2-g_{K}
$$

By Lemma 6 (c),

$$
f_{K}^{\prime}=f_{K}+1
$$

Moreover, there is a unique vertex in $K^{\prime}$ for any vertex in $V(K) \backslash V(P l(K))$ and the vertices from $P l(K)$ are replaced by the vertices of $c$. Hence

$$
n_{K}^{\prime}=n_{K}-n_{P l}+|c| .
$$

Similarly, for the number of edges we have

$$
m_{K}^{\prime}=m_{K}-m_{P l}+|c| .
$$

Combining the last three equalities with (7) and (8) we get

$$
\begin{aligned}
n_{K}^{\prime}-m_{K}^{\prime} & +f_{K}^{\prime}=\left(n_{K}-m_{K}+f_{K}\right)-\left(n_{P l}-m_{P l}\right)+1 \\
& =\left(2-2 g_{K}\right)-\left(2-2 g_{K}\right)+2=2 .
\end{aligned}
$$

Hence, by $(2), \bar{\mu}\left(K^{\prime}\right)$ is planar. 
3.3.3 Transforming $\bar{\mu}\left(K^{\prime}\right)$ into a planar drawing of $K$ with a small crossing number. Recall that the cycle $c$ in $K^{\prime}$ corresponds to the subgraph $P l(K)$ of $K$. Replacing $c$ with $P l(K)$ will transform $K^{\prime}$ back into $K$.

Without loss of generality assume that the face corresponding to $c$ is not the outer face. Remove all vertices from $c$ and all of their incident edges. Denote by $h$ the resulting face. Draw all vertices of $P l(K)$ inside $h$. By Lemma 6 (b), all edges of $K$ incident to a vertex in $P l(K)$ will have both their endpoints inside $h$. Since there are no more than $d n_{P l}$ such original edges, they can be drawn inside $h$ with no more than $\left(d n_{P l}\right)^{2}$ original crossings.

By Lemma 6 (a), the above operation transforms the embedding of $K^{\prime}$ into a drawing of $K$. Let $\bar{\mu}(K)$ denote the resulting drawing. We summarize the properties of that drawing in the following lemma.

Lemma 8. $\bar{\mu}(K)$ is a drawing of $K$ in the plane that has no more than $\left(d n_{P l}\right)^{2}$ original crossings.

3.3.4 Routing the stubs of $K$ to the outer face. Assign length 0 to all edges joining two vertices on level $l_{K}^{-}$and assign length 1 to all other edges of $K$. A length of a path in $K$ is defined as the sum of the lengths of its edges. We will make use of the following fact.

Lemma 9. Between any pair of vertices of $K$ there exists a path entirely in $K$ of length no more than $2 q_{K}(r-1)+q_{K}-1$.

Proof. Let $Q_{1}, \cdots, Q_{q_{K}}$ be all bicomponents induced by the set of the vertices on level $l_{K}^{-}$. Let $v$ and $w$ be any two vertices of $K$. Construct a path $p=\left(v_{1}, \cdots, v_{s}\right)$ in $K$ joining $v$ and $w$. Suppose $v$ is a descendant of a vertex from $Q_{i}$. Let $j$ be the largest index for which $v_{j}$ is a descendant of a vertex from $Q_{i}$. Replace the subpath $\left(v_{1}, \cdots, v_{j}\right)$ with a simple path $p_{1}$ that uses forest edges only. The length of $p_{1}$ is no greater than $2(r-1)$. By the choice of $j$, the path $\left(v_{j+1}, \cdots, v_{s}\right)$ has no vertices that are descendants of a vertex from $Q_{i}$. Using induction, the subpath $\left(v_{j+1}, \cdots, v_{s}\right)$ can be replaced by a path $p_{2}$ between $v_{j+1}$ and $v_{s}$ of length no more than $2\left(q_{K}-1\right)(r-1)+q_{K}-2$. Merging $p_{1}$, the edge $\left(v_{j}, v_{j+1}\right)$, and $p_{2}$ results in a path between $v$ and $w$ of length at most $2 q_{K}(r-1)+q_{K}-1$.

Finally, route all stubs of $K$ to the outer face of $\bar{\mu}(K)$ using Lemma 9. More precisely, let $s$ be a stub corresponding to an original edge and let $p$ be the path constructed by the procedure of Lemma 9 for $s$. Informally, $s$ will be routed along a path "parallel" to $p$ that avoids vertices of $p$ and that, for the portions of the path on level $l_{K}^{-}$, makes a "shortcut" inside the corresponding faces in order to minimize the number of intersections. More formally, remove all edges incident to vertices of $p$ on levels greater than $l_{K}^{-}$(i.e., that have lengths 1 ) or on level $l_{K}^{-}$, but not on $p$. This operation creates a new face $f$ that includes all faces defined by the vertices on level $l_{K}^{-}$and that have at least one edge from $p$. Route $s$ inside $f$ avoiding vertices from $p$. Then $s$ will intersect no more than $l(s) \cdot d$ original edges of $G$ plus a number of stubs of $K$. (We will separately bound the number of all intersecting pairs of original stubs in our analysis below.)

Recall that $L_{K}=N(V(K)) \cap L_{i^{*}}$, where $L_{i^{*}}$ was defined in (3). The next lemma summarizes the results of this section regarding the drawing of $K$.

Lemma 10. The constructed drawing of $K$ has less than $8\left(d g_{K} r\right)^{2}+2 w t\left(L_{K}\right)^{2}+2 d q_{K} r$ original crossings.

Proof. Crossings in the embedding of $K$ may have occurred during the planarizing step (Subsection 3.3.3), or from stubs routed to the outer face. By Lemma 8, the original crossings from the first type are no more than $\left(d \cdot n_{P l}\right)^{2}$, which by Lemma 5 is no more than $\left(2 d g_{K}(r-1)+w t\left(L_{K}\right)\right)^{2}<$ $8\left(r d g_{K}\right)^{2}+2 w t\left(L_{K}\right)^{2}$, where the $2 w t\left(L_{K}\right)^{2}$ bound accounts for all possible intersections between pairs of original stubs. By Lemma 9, the number of original crossings of the second type are no more than $d\left(2 q_{K}(r-1)+q_{K}-1\right)<2 d q_{K} r$, excluding crossing between pairs of original stubs, which have already been counted (we assume that no two edges cross more than once).

When we apply the above algorithm to all non-planar components, the total number of original crossings is estimated in the following lemma. 
Lemma 11. All non-planar components of $G$ can be drawn in the plane so that the unattached endpoints of all stubs are in the outer face of the drawing and the total number of original crossings is no more than $8(d g r)^{2}+12 d g r+2(m / r)^{2}$.

Proof. By Lemma 10 the total number of original crossings is bounded by

$$
\begin{gathered}
\sum_{K \text { is } l-p l a n a r}\left(8\left(r d g_{K}\right)^{2}+2 w t^{2}\left(N(V(K)) \cap L_{i^{*}}\right)+2 d q_{K} r\right) \\
\leq 8(d g r)^{2}+12 d g r+2 w t^{2}\left(E(G) \cap L_{i^{*}}\right) \quad \text { (by Lemma 4) } \\
\leq 8(d g r)^{2}+12 d g r+2(m / r)^{2} \quad \text { (by (3)). }
\end{gathered}
$$

\subsection{Drawing s-planar components}

This case is similar to the case of non-planar components, except that there is no need to planarize.

Lemma 12. All s-planar components of $G$ can be drawn in the plane so that the unattached endpoints of all stubs are in the outer face and the total number of original crossings is no more than $12 d g r$.

Proof. Let $K$ be any s-planar component. Draw $K$ in the plane (with 0 crossings) so that one of the cycles defined by the vertices on the lowest level is the outer face. Route all stubs of $K$ to the outer face of the drawing as described in the proof of Lemma 9. By Lemma 9, the drawing of $K$ has less than $2 d q_{K} r$ original crossings.

Draw by the same procedure all remaining s-planar components. By Lemma 4, the total number of crossings for all s-planar components is no more than

$$
\sum_{K \text { is }} 12 d q_{K} r \leq 12 d g r .
$$

\subsection{Drawing l-planar components}

Since l-planar components may have up to $\Omega(n)$ levels, the bound derived from (3) on the number of edges that have to be routed is not sufficient to guarantee a small crossing number. Hence, we have to additionally cut each l-planar component along some small set of edges joining two consecutive levels.

Let $K$ be any l-planar component and let $l_{K}^{-}$and $l_{K}^{+}$be the lowest and the highest levels of $K$, respectively. By Lemma 1, the embedding of $K^{-}$induced by the embedding of $G$ is planar.

Denote by $f^{-}$and $f^{+}$the faces defined by the set of the vertices on levels $l_{K}^{-}$and $l_{K}^{+}$, respectively. We will make use of the following fact.

Lemma 13. Let $v$ be a vertex on level $i$, where $l_{K}^{-} \leq i \leq l_{K}^{+}$. There exists a continuous line in the plane joining $v$ to a point inside $f^{-}$(respectively $\left.f^{+}\right)$that contains no other vertices of $K$ except $v$ and with no more than $\left(i-l_{K}^{-}+1\right) d$ (respectively $\left(l_{K}^{+}-i+1\right) d$ ) original crossings with edges from $K$, excluding the edges joining a vertex on level $i$ and a vertex on level $i+1$.

Proof. Suppose $a$ is any point inside $f^{+}$. Let $x$ be any vertex on level $l_{K}^{+}$. There exists a path $p$ of $i-l_{K}^{-}+1$ vertices joining $x$ with some vertex, say $w$, on level $i$. The set $S$ of all original edges incident to vertices of $p$ has size no more than $\left(i-l_{K}^{-}+1\right) d$. Moreover, the removal of all the edges of $S$ creates a new face $q$ that contains both $w$ and $a$ inside it. Let $l_{1}$ be a line joining $w$ and $a$ that lies entirely inside $f$. Clearly, the set of the edges of $G$ that $l_{1}$ intersects is a subset of $S$ and hence $l_{1}$ can be chosen so that it has no more than $\left(i-l_{K}^{-}+1\right) d$ original crossings with such edges. Furthermore, $w$ and $v$ can be connected by a line $l_{2}$ that intersects only edges joining vertices on levels $i$ and $i+1$. Connect $l_{1}$ and $l_{2}$ at $w$ and modify the joined line so that it avoids $w$. The resulting line satisfies the lemma. The case where $a$ is inside $f^{-}$is similar. 
Lemma 14. There exists a drawing of $K$ in the plane with at most $|V(K)|+w t\left(L_{K}\right)^{2}$ original crossings such that all stubs have their unattached endpoints drawn in the outer face.

Proof. Find a level $i$ such that the number of the original edges of $K$ joining a vertex on level $i$ with a vertex on level $i+1$ is minimum. Replace each edge joining levels $i$ and $i+1$ by a pair of stubs as we did with $G$ in Section 3.1. This splits $K$ into components $K^{-}$, induced by the vertices in $K$ on levels less than or equal to $i$, and $K^{+}$, induced by the vertices in $K$ on levels greater than $i$.

Convert $\mu\left(K^{-}\right)$into a drawing with an outer face $f^{-}$. For any stub $s$ of $K^{-}$incident to a vertex from level $i$, use Lemma 13 to route $s$ to $f^{-}$so that $s$ intersects no more than $\left(i-l_{K}^{-}+1\right) d$ edges of $K^{-}$. Repeat the procedure for all other stubs of $K^{-}$incident to vertices from level $i$. The total number of original crossings produced in this step is no more than $L(i)\left(i-l_{K}^{-}+1\right) d \leq\left|V\left(K^{-}\right)\right| d$, where $L(i)$ denotes the number of original edges joining a vertex on level $i$ and a vertex on level $i+1$.

Similarly, convert $\mu\left(K^{+}\right)$into a drawing with an outer face $f^{+}$and route all stubs of $K^{+}$incident to vertices from level $i+1$ to $f^{+}$, producing no more than $\left|V\left(K^{+}\right)\right| d$ original crossings. Add to the drawing the one of $K^{-}$found in the previous step. Finally, merge any pair of stubs incident to vertices from levels $i$ and $i+1$ into their parent edge of $G$. Since all stubs were already in the outer face of the drawing, all new intersections are between stubs incident to vertices from levels $i$ and $i+1$. Hence the number of new original crossings is no more than $(L(i))^{2}$, resulting in a total number of original crossings no more than

$$
\left|V\left(K^{-}\right)\right| d+\left|V\left(K^{+}\right)\right| d+(L(i))^{2}=|V(K)| d+(L(i))^{2} \leq|V(K)| d+w t^{2}\left(L_{K}\right) .
$$

We summarize the results of this section in the following lemma.

Lemma 15. All l-planar components can be drawn in the plane so that the unattached endpoints of all stubs are in the outer face of the drawing and the total number of original crossings is no more than $n d+(m / r)^{2}$.

Proof. Apply Lemma 14 to all components of l-planar type. The total number of original crossings for all components of that type is

$$
\begin{gathered}
\sum_{K \text { is } l-p l a n a r}\left(|V(K)| d+w t^{2}\left(N(V(K)) \cap L_{i^{*}}\right)\right) \leq|V(G)| d+w t^{2}\left(E(G) \cap L_{i^{*}}\right) \\
\leq n d+(m / r)^{2} \quad(\text { by }(3)) .
\end{gathered}
$$

\subsection{Reconnecting the embedded components}

After all components of $G-S$ are drawn in the plane by applying the algorithms described in Subsections $3.3,3.4$, and 3.5, all the $w t\left(L_{i^{*}}\right)$ original stubs will have their unattached endpoints in the outer face. Joining all pairs of original stubs into their parent edges so that no two stubs intersect more than once will produce at most $w t\left(L_{i^{*}}\right)^{2}$ additional original crossings. This leads to the following result.

Theorem 1. Any n-vertex graph of maximum degree d embedded in $S_{g}$ can be drawn in the plane with $O(d g n)$, if $g=o(n)$, or $O\left(g^{2}\right)$, if $g=\Omega(n)$, edge crossings.

Proof. By Lemmas 11, 12, and 15, the total number of original crossings from drawing individual components is no more than

$$
8(d g r)^{2}+24 d g r+n d+3(m / r)^{2}=O\left((d g r)^{2}+n d+3(m / r)^{2},\right.
$$

where $m$ is the number of original edges of $G$. Choosing $r=\lceil\sqrt{m /(g d)}\rceil$ and adding the number of the original crossings resulting from joining the stubs in the final step, which is bounded by $w t^{2}\left(L_{i^{*}}\right)=$ $O\left((m / r)^{2}\right)$, the number of all original crossings is $O(d g m+d n)=O(d g m)$. 
Without loss of generality we can assume that $G$ is connected and has no vertex of degree two. Then we have the inequalities $m \geq 3 / 2 n$ and $m \geq 3 / 2 f$, which we will use next, where $f$ is the number of the faces of the embedding.

If $g=o(n)$, then from the Euler formula (2) and the above inequalities $m=O(n)$ and hence $O(d g m)=O(d g n)$, which proves the theorem. If $g=\Omega(n)$, then by $(2) m=\Omega(n)$ and $g=\Omega(m)$. Since any straightline drawing of $G$ in the plane has less than $m^{2}=O\left(g^{2}\right)$ crossings, it satisfies the theorem.

Corollary 2. Let $G$ be any $n$-vertex bounded degree graph and let $0<g=o(n)$. Then

$$
\operatorname{cr}(G)=O\left(\operatorname{cr}_{g}(G) g+g n\right) .
$$

Proof. Draw $G$ in $S_{g}$ with $\mathrm{cr}_{g}(G)$ crossings. Replace every crossing by a new vertex. We get a new bounded degree graph $G^{\prime}$ of genus $g$ and $\operatorname{cr}_{g}(G)+n$ vertices. By Theorem 1 we have

$$
\operatorname{cr}\left(G^{\prime}\right)=O\left(\left(\mathrm{cr}_{g}(G)+n\right) g\right) .
$$

Observe that $\operatorname{cr}(G) \leq \operatorname{cr}\left(G^{\prime}\right)+\operatorname{cr}_{g}(G)$, which implies the result.

\subsection{Complete algorithm and complexity analysis}

Here we describe the entire algorithm and analyze its complexity.

\section{Algorithm DRAW}

Input: An $n$-vertex, $d$-degree graph $G$, an embedding $\mu(G)$ of $G$ in $S_{g}$. Output: A drawing of $G$ with $O\left(\max \left\{d g n, g^{2}\right\}\right)$ crossings.

1. If $g=\Omega(n)$, construct an arbitrary straightline drawing of $G$ and exit.

2. Triangulate $\mu(G)$ assigning weight 0 to any new edge and weight 1 to any original edge of $G$.

3. Set $r=\lceil\sqrt{|E(G)| /(g d)}\rceil$. Divide the vertices of $G$ into levels depending on their distances to a chosen vertex. Cut a subset of selected edges joining consecutive levels, as described in Section 3.1, producing components of the following three types: (i) non-planar components, having at most $r$ levels and induced genus greater than zero; (ii) s-planar components, having at most $r$ levels and genus 0; and (iii) l-planar components, whose vertices on their lowest and on their highest levels define single faces and that have genus 0 . Each cut edge is replaced by a pair of stubs.

4. For each component $K$, draw $K$ in the plane applying one of the Steps 5, 6, or 7 .

5. If $K$ is non-planar, then

5.1. Construct a subgraph $P l(K)$ of $K$ such that (i) $P l(K)$ contains at most $2 g_{K}(r-1)$ vertices not counting the vertices on the highest and the lowest levels of $K$; (ii) converting $P l(K)$ into a simple cycle $c$ that is a face, denoted by $f$, of the new embedding as described in Section 3.3.2 transforms the embedding of $K$ into an embedding of the updated graph, denoted by $K^{\prime}$, in $S_{0}$. Moreover, $P l(K)$ and $c$ have the same set, $M$, of edges joining them to $K$ and $K^{\prime}$, respectively. 5.2. Draw $K^{\prime}$ in the plane with $f$ as an outer face.

5.3. In order to transform $K^{\prime}$ back to $K$, remove $c$, draw the vertices of $P l(K)$ in the resulting face, $f^{\prime}$, and draw all edges of $M$. Since both endpoints of any edge from $M$ are on or inside $f^{\prime}$, intersections will occur only between pairs of edges from $M$.

5.4. Route any stubs of $K$ to the infinite face of the drawing as described in the proof of Lemma 9 and join matching pairs of stubs into their parent edges.

6 . If $K$ is s-planar, then draw $K$ in the plane with one of the cycles determined by the vertices on the lowest level of $K$ as outer face and continue as in Step 5.4.

7. If $K$ is l-planar, choose a pair of adjacent levels of $K$ such that the total weight of the edges between vertices on those levels is minimum. Replace these edges by pairs of stubs and draw the resulting planar components on the plane so that the cycles on the lowest and on the highest level of $K$ are outer faces. Route each stub to the corresponding outer face as in the proof of Lemma 9. Merge all matching pairs of stubs with both endpoints in $K$ into their parent edges. 
8. After all components are drawn in the plane, restore $G$ by merging matching pairs of the remaining stubs (all located in the infinite face) into their parent edges.

It is easy to show that Steps 1,2 , and 3 can be implemented in $O(|G|)$ time. Steps 5.1 and 5.2 take $O(|K|)$ time, and Steps 5.3, 5.4, 6, and 7 can be implemented in time proportional to the number of the original crossings produced in these steps. Hence we have the following.

Theorem 2. Algorithm DRAW constructs a drawing satisfying Theorem 1 of any n-vertex d-degree graph embedded in $S_{g}$ in $O(d g n)$ time, if $g=o(n)$, or in $O\left(g^{2}\right)$ time, if $g=\Omega(n)$.

\section{Lower bound}

In this section we provide a matching lower bound for Theorem 1.

Theorem 3. There is a constant $\alpha$ such that for any positive integers $n, g<n^{2}$, and $d<n$, there exists an $n$-vertex graph $G$ of genus and degree not exceeding $g$ and $d$, respectively, such that

$$
\operatorname{cr}(G) \geq \alpha d g n \text {, if } g=o(n) \text {, or } \operatorname{cr}(\mathrm{G}) \geq \alpha \mathrm{g}^{2} \text {, otherwise. }
$$

Proof. Let $g, d$, and $n$ be any integers such that $g=O\left(n^{2}\right)$ and $d=O(n)$. We will show that there exists a graph of $\bar{n}=\Theta(n)$ vertices, degree $\bar{d}=\Theta(d)$, genus $\bar{g}=\Theta(g)$, and crossing number satisfying (9) for some global constant $\alpha$.

It is known $[16,17]$ that any $n$-vertex graph $H$ satisfies

$$
\operatorname{cr}(H) \geq \frac{1}{40} \mathrm{bw}^{2}(H)-\frac{1}{16} \sqrt{\sum_{v \in V(H)} \operatorname{deg}^{2}(v) .}
$$

Consider the following two cases.

(a) $g=o(n)$. In [18], it was a shown that for any $g, n$, and $d$, there exists an $\bar{n}$-vertex, $\bar{d}$-degree, $\bar{g}$-genus graph $G$, such that $\bar{n}=\Theta(n), \bar{d}=\Theta(d), \bar{g}=\Theta(g)$, and

$$
\mathrm{bw}(G) \geq \beta \sqrt{\bar{d} \bar{g} \bar{n}},
$$

for some absolute constant $\beta$. Suppose $G$ is such a graph. By (10),

$$
\operatorname{cr}(G)=\Omega(\bar{d} \bar{g} \bar{n})-O(\bar{d} \bar{n})=\Omega(\bar{d} \bar{g} \bar{n}) .
$$

(b) $g=\Omega(n)$. Let $s=\lceil\sqrt{g}\rceil$ and $G$ be a graph consisting of the complete graph on $s$ vertices $K_{s}$ plus $\max \{0, n-s\}$ isolated vertices. Clearly, $G$ has $\bar{n}=\max \{n, s\}=\Theta(n)$ vertices, genus $\bar{g}=\Theta\left(s^{2}\right)=\Theta(g)$, and crossing number

$$
\operatorname{cr}(G)=\operatorname{cr}\left(K_{s}\right)=\Theta\left(s^{4}\right)=\Theta\left(g^{2}\right) .
$$

Since $g=\Omega(n)$, then $\bar{g}=\Omega(\bar{n})$ and the theorem follows.

\section{$5 \quad$ Extensions and generalizations}

The bound in Theorem 3 on the time complexity of our algorithm is asymptotically optimal, if the algorithm is required to explicitly output all edge crossings. However, if a succinct encoding of the output is allowed, then a more careful implementation of the drawing phase of the algorithm can reduce the complexity to $O(|G|)$. For instance, instead of listing all intersections of an edge $e$ with the edges incident to the vertices of a path $\left(v_{0}, \cdots, v_{k}\right)$ such that $v_{i}$ is a parent of $v_{i-1}$ in $F_{K}$, for $1 \leq i \leq k$, we need only to output the endpoints $v_{0}$ and $v_{K}$, since they uniquely determine that portion of the drawing. If $g=\Omega(n)$, then we need only to output the positions of the vertices, since they determine 
the drawing satisfying the theorem (the edges will be drawn as straight-line segments). We omit the detailed prescription of such an algorithm here for a lack of space.

Pach and Toth showed in [15] that their $O\left(c^{g} d n\right)$ upper bound on the crossing number of $n$-vertex $d$-degree graphs of genus $g$ can be generalized to graphs of arbitrary degrees by substituting the product $d n$ in their bound by the number $\sigma(G)=\sum_{v \in V(G)} \operatorname{deg}^{2}(v)$. The technique they use for this purpose is to replace any vertex $v$ of $G$ by a $\operatorname{deg}(v) \times \operatorname{deg}(v)$ 2-dimensional mesh $M_{v}$ of vertices, where the edges incident to $v$ appear in the same order around the boundary of $M_{v}$ as they do around $v$. Clearly, the same technique, if used to modify the proof of our Theorem 1, will result in an upper bound of $O(\sigma(G) g)$ for the case $g=o(n)$. Such an approach, however, will increase the size of the graph, and hence the computation time, by a factor of $\sigma(G) /|G|$ (if a succinct encoding of the output is used), which can be as much as $\Omega(n)$. This increase in time can be reduced to only $O(\log n)$, while achieving the same bound on the crossing number, by using the technique from [6] that is based on shortest path computation in a modified version of $G$.

\section{References}

1. M. Ajtai, V. Chvátal, M. M. Newborn, E. Szemerédy. Crossing-free subgraphs. Theory and Practice of Combinatorics, North Holland Mathematical Studies 60, Annals of Discrete Mathematics 12, North-Holland, Amsterdam, 1982, 9-12.

2. C. Buchheim, D. Ebner, M. Jünger, G. W. Klau, P. Mutzel, R. Weiskircher. Exact crossing minimization. 13th Intl. Symposium on Graph Drawing, Lecture Notes in Computer Science, Springer, Berlin, 2006.

3. G. Di Battista, P. Eades, R. Tamassia, I.G. Tollis. Graph Drawing: Algorithms for Visualization of Graphs. Prentice Hall, 1999.

4. R. Cimikowski. Algorithms for the fixed linear crossing number problem. Discrete Applied Mathematics 122:93-115, 2002.

5. H. N. Djidjev. A separator theorem. Compt. rend. Acad. bulg. Sci., 34:643-645, 1981.

6. H. N. Djidjev. Partitioning planar graphs with vertex costs: Algorithms and applications. Algorithmica, 28(1):51-75, 2000.

7. H. N. Djidjev, S. Venkatesan. Planarization of graphs embedded on surfaces. 21st International Workshop on Graph-Theoretic Concepts in Computer Science, pages 62-72, 1995.

8. G. Even, S. Guha, B. Schieber. Improved Approximations of Crossings in Graph Drawings and VLSI Layout Areas. SIAM J. Computing, 32(1): 231-252, 2002.

9. M. R. Garey, D. S. Johnson. Crossing number is NP-complete SIAM J. Algeraic and Discrete Methods, 1983:4, 312-316.

10. J. R. Gilbert, J. P. Hutchinson, R. E. Tarjan. A separator theorem for graphs of bounded genus. J. Algorithms, 5:391-407, 1984.

11. L.Y. Glebsky, G. Salazar. The crossing numbert of $\operatorname{cr}\left(C_{m} \times C_{n}\right)$ is as conjectured for $n \geq m(m+1)$. J. Graph Theory 47:53-72, 2004.

12. P. Hliněný. Crossing number is hard for cubic graphs. Mathematical Foundations of Computer Science, Lecture Notes in Computer Science 3153, Springer, Berlin, 772-782, 2004.

13. F.T. Leighton. Complexity Issues in VLSI. M.I.T. Press, Cambridge, 1983.

14. A. Liebers, A. Methods for planarizing graphs - a survey and annotated bibliography. J. of Graph Algorithms and Applications 5:1-74,2001.

15. J. Pach, G. Tóth. Crossing number of toroidal graphs. 13th Intl. Symposium on Graph Drawing, Lecture Notes in Computer Science 3843, Springer, Berlin, 334-342, 2006.

16. J. Pach, F. Shahrokhi, M. Szegedy. Applications of the crossing number. Algorithmica, 16:111-117, 1996.

17. O. Sýkora, I. Vrt'o. Optimal VLSI Layouts of the star graph and related networks. Integration the VLSI Journal, 17:83-94, 1994.

18. O. Sýkora, I. Vrt'o. Edge separators for graphs of bounded genus with applications. Theoretical Computer Science, 112:419-429, 1993.

19. I. Vrt'o. Crossing numbers of graphs: A bibliography. Available electronically at http://www.ifi.savba.sk/imrich/.

20. K. Zarankievicz. On a problem of P. Turán concerning graphs. Fund. Math., 41:137-145, 1954. 